\title{
Abordaje interdisciplinar para la enseńanza de las ciencias y la actualización de profesores
}

\section{MARÍA GABRIELA LORENZO}

Universidad de Buenos Aires. Facultad de Farmacia y Bioquímica. Centro de Investigación y Apoyo a la Educación Científica (CIAEC). Instituto de Investigación en Educación Superior (IIES). Consejo Nacional de Investigaciones Científicas y Técnicas (CONICET). Ciudad de Buenos Aires, Argentina.

glorenzo@ffyb.uba.ar

Recibido8 de julio 2020 - Aceptado 30 de octubre2020

\section{Resumen}

En este trabajo se propone una revisión del enfoque interdisciplinar orientado a la construcción del conocimiento científico, didáctico y docente, como una alternativa para la revisión de las prácticas educativas y la enseñanza de las ciencias naturales. Se revisan algunos aportes de la literatura, centrando la atención en los enfoques CTS y STEM, y su impacto en la resolución de cuestiones sociocientíficas. Se presenta y discute una propuesta de actividad experimental simple, que fue implementada en diferentes dispositivos de enseñanza, con el fin de ilustrar la versatilidad de estos enfoques para la diversificación de la enseñanza. Se exponen las conexiones entre dichos ejemplos, el campo de la investigación en didáctica de las ciencias y la formación de los profesores.

Palabras clave: ciencia-tecnología-sociedad, STEAM, formación docente, investigación científica.

\begin{abstract}
In this work, a review of an interdisciplinary approach is proposed in order to foster the construction of the educational, pedagogical and scientific knowledge, as an alternative to examine the educative practices and natural science teaching. Some contributions from literature were reviewed paying attention to STS and STEM approaches and their impact on socio-scientific issues. In order to show the versatility of these approaches to think about a varied way of teaching, a simple practical activity, which was carried out in different teaching situations, is presented and discussed.The connections among those examples, the science education research and teachers' education, are exposed.
\end{abstract}

Key words: science-technology-society, STEAM, teacher education, scientific research. 


\section{Introducción}

La preocupación de las sociedades de nuestro tiempo por lograr una formación científica de calidad para sus ciudadanos es un tema permanente en la agenda de todos los países. Esta formación enfrenta dos cuestiones aparentemente difíciles de conciliar: la alfabetización científica de todas las personas y la formación disciplinar básica que les permita a los egresados de la educación secundaria, proseguir sus estudios en carreras vinculas a las ciencias naturales y experimentales. En este sentido, los enfoques interdisciplinares han mostrado ser una estrategia útil para zanjar esta brecha, no solo en cuestiones vinculadas a la enseñanza de las ciencias sino también en lo que respecta al diseño de propuestas de capacitación para el colectivo docente. Es por eso que, resulta imprescindible promover el análisis y la reflexión sobre el impacto de la interdisciplinariedad como una nueva forma de abordar la enseñanza de las ciencias naturales en relación con los aportes de la investigación en didáctica de las ciencias que promuevan el trabajo colaborativo a la vez que ofrezcan un espacio propicio para la socialización de la producción científica y académica.

En cuanto a la interdisciplinariedad es considerada actualmente como una tendencia y una posibilidad que atraviesa diversos intereses educativos. Por un lado, como un nuevo marco para repensar la enseñanza; $y$, por otro, como una necesidad para desarrollar la investigación en el campo de la didáctica de las ciencias. Consecuentemente, los objetivos de este trabajo son:

- Revisar algunos enfoques actuales para la enseñanza de las ciencias naturales basados en la interdisciplinariedad.

- Presentar algunos ejemplos de prácticas de enseñanza diseñadas a partir de dichos enfoques que fueron aplicadas en cursos de capacitación de profesores de ciencias naturales.

- Hacer visibles las conexiones entre dichos ejemplos y el campo de la investigación en didáctica de las ciencias.

En definitiva, tomando como eje la interdisciplinariedad, se discute su potencialidad como estrategia para la enseñanza, para la investigación educativa y su aplicación en la formación docente y en la capacitación de profesores.

\section{Cuando con una única disciplina no es suficiente}

Para comenzar a hablar acerca de la interdisciplinariedad como un enfoque alternativo tanto para la enseñanza como para la investigación educativa, es necesario revisar algunas ideas con el fin de acordar los significados de modo que nos permita lograr una comunicación efectiva en una comunidad dada de aprendizaje (Jones, Gardner, Robertson \& Robert, 2013, Lantz-Andersson, Lundin \& Selwyn, 2018).

Cuando hablamos de una disciplina nos estamos refiriendo a un campo específico de conocimiento, a un dominio en particular, que incluye un conjunto de modelos, teorías y conceptos, así como un vocabulario técnico específico, determinadas estrategias metodológicas, fines, ideales, puntos de vista, que son compartidos por las personas que se desempeñan en dicho campo tanto como investigadores científicos, profesionales o docentes. Es decir que cada disciplina constituye una manera particular de abordar los problemas. Así, la química, la física, la biología, en el área de las ciencias naturales, y la historia, la sociología o la economía, en el área de las ciencias sociales, son disciplinas ya que se ocupan de un dominio particular de conocimiento, cada una con su propia naturaleza y toda actividad que se vincule a cada una de ellas será clasificada como disciplinar.

Sin embargo, existen ciertas situaciones que se desarrollan en los límites entre dos disciplinas, que finalmente se constituyen en disciplinas consolidadas, con un corpus de conocimiento robusto, por ejemplo la fisicoquímica, la bioquímica o la biofísica, por mencionar algunas. En esta línea, también podría incluirse a la didáctica de las ciencias. Y más recientemente, han surgido nuevos enfoques que intentan no restringir el análisis de un objeto o fenómeno a una única disciplina, sino que por el contrario, consideran que la complejidad del mundo natural y social que nos rodea, no puede ser estudiado desde una sola perspectiva, sino que se requieren los aportes de una diversidad de disciplinas (Peñuela Velásquez, 2005). Desde esta perspectiva la interdisciplina aparece como una concurrencia de modelos teóricos y metodológicos para abordar problemas concretos, los cuales con el correr del tiempo, pueden dar lugar al surgimiento de nuevas disciplinas.

Es así que se incorporan al lenguaje una serie de nuevos términos, a partir de la utilización de prefijos latinos al vocablo disciplinar que intentan definir estos nuevos puntos de vista. 
- Multidisciplinariedad: Surge de incluir el prefijo multi, que significa muchos, refiere a una gran cantidad de objetos, que pueden o no guardar semejanzas entre sí, por ejemplo: multivitamínico. De este modo, la multidisciplinariedad se define como el conjunto de disciplinas que se proponen simultáneamente para resolver un problema o estudiar una situación, pero sin que se hagan explícitas las relaciones que pueden existir entre ellas. Es decir, que cada disciplina aborda el problema a situación de manera independiente de las otras, pudiendo haber o no una comunicación entre ellas.

- Pluridisciplinariedad: Este concepto, si bien se parece al anterior, se diferencia por la utilización del prefijo pluri, que hace referencia a la existencia de varios objetos (que pueden ser muchos o pocos) pero que se caracterizan por ser diferentes entre sí, refiere entonces a variedad. Consecuentemente, la pluridisciplinariedad puede definirse como la yuxtaposición de disciplinas diversas que se sitúan generalmente en el mismo nivel jerárquico y se agrupan de manera tal que se subrayen las relaciones que existen entre ellas.

- Interdisciplinariedad: En este caso se recurre al uso del prefijo latino inter cuyo significado da cuenta de algo que se ubica en medio de o dentro de al menos otras dos cosas, es aquello que está entre diversos objetos o fenómenos, como cuando nos referimos a los tratados internacionales, o los viajes interplanetarios. En este sentido, podemos definir a la interdisciplinariedad como el conjunto de disciplinas conectadas entre sí y con relaciones definidas, entre las que se establece una relación dialéctica a fin de que, el resultado del desarrollo conjunto de sus actividades conduzca a un producto o idea común.

- Transdisciplinariedad: Recientemente también se ha incluido la utilización del prefijo trans (o tras) cuyo significado principal es transmitir la idea de cambio, algo que va más allá, como los buques transocéanicos. Consecuentemente puede definirse la transdisciplinariedad como la formulación de problemas y de propuestas para comprenderlos y resolverlos, mediante la interacción de especialistas de diversas disciplinas, así como de agentes que no provienen de ninguna disciplina pero que pueden hacer aportes de conocimientos relevantes. De manera tal que el producto final trasciende cualquier resultado obtenido de manera individual (Olivé Morett, Argueta Villamar \& Puchet Anyul, 2018).
Desde nuestro punto de vista, la didáctica de las ciencias podría considerarse comprendida en esta categoría ya que su carácter metadisciplinario se nutre de diversos dominios de conocimiento.

Sin embargo, cabe aclarar que si bien estas palabras resultan aparentemente muy semejantes entre sí, encierran diferencias sustanciales a la hora de pensar en los enfoques para la enseñanza y por tanto, en las estrategias que se desarrollen para concretarlos, como veremos más adelante.

\section{La interdisciplinariedad como estrategia para la enseñanza}

Entre las razones que esgrimen diversos autores sobre la importancia de los enfoques interdisciplinares (Bolarín Martínez, Moreno Yus \& Porto Currás, 2013, Carvajal Escobar, 2010), rescatamos los siguientes:

El enfoque interdisciplinar toma como objeto de trabajo el estudio de problemas reales y contextualizados y que por lo tanto resultan próximo a los conocimientos e intereses de los estudiantes (Llano Arana et al., 2016). De este modo, se estimulan las vocaciones científicas de los jóvenes para el estudio de la ciencia y de la tecnología, a través de una renovación en la educación de las ciencias y la alfabetización científica de la ciudadanía. Hecho que se considera altamente relevante en la actualidad debido al descenso de las matrículas en las carreras de corte científico, en diversos países sin distinción de fronteras.

Las propuestas que consideran el enfoque interdisciplinar no son nuevas, por ejemplo en su trabajo Lenoir (2013) recorre treinta años de su aplicación en la educación primaria destacando la importancia de la búsqueda de puntos de convergencia entre las disciplinas, por sobre sus diferencias. También encontramos propuestas para la educación secundaria (Castañer BaIlcells \& Trigo Aza (1995). Los problemas ambientales dada su naturaleza sistémica y compleja, han sido unos de los promotores del enfoque interdisciplinario para su estudio en contextos educativos Palma de Arraga (1998). Algo más adelante, la mirada interdisciplinaria fue tomada desde el modelo de competencias para promover la autonomía de los estudiantes en el nivel superior (Posada Álvarez, 2004). En esta línea, es interesante recorrer los diversos procesos de organización escolar, estrechamente ligados (a favor o en contra) con las políticas económicas como lo recoge Torres Santomé (1994) al analizar las características del currícum integrado en un mundo marcado por la globalización. 
Dada la urgencia de resolver problemas en el que aparecen varios aspectos a ser considerados y estudiados, este enfoque impulsa el desarrollo de las competencias científicas, computacionales y tecnológicas necesarias para las necesidades del mundo actual. Y también, promueve un conjunto de habilidades diferentes de las que fomenta la enseñanza tradicional, dado que incluyen el trabajo en grupo y las habilidades comunicativas, entre otras.

La didáctica de las ciencias naturales ha producido a lo largo de los últimos treinta años, dos enfoques de enseñanza basados explícitamente en la interdisciplinariedad: el modelo STEAM (o STEM) y el enfoque CTS.

El modelo STEAM hace referencia a un enfoque que contempla de manera conjunta a las ciencias (Sciences), la tecnología (Technology), la ingeniería (Engineering), el arte (Arts) y la matemática (Mathematics) (Santillán Aguirre, Cadena Vaca \& Cadena Vaca, 2019). En los casos en que el arte no es considerado dentro de la propuesta el enfoque se denomina STEM. Desde esta perspectiva, las ciencias son concebidas en un sentido amplio incluyendo tanto las denominadas naturales como las sociales y humanas. Los aportes de la tecnología están más bien orientados a las que se conocen como tecnologías de la comunicación, la informática y la programación. Mientras que a la producción de objetos concretos (maquetas, robots, diseños) se los incluye dentro de la ingeniería. Y, tanto el arte como las matemáticas, estarían permeando diferentes aspectos y momentos de las tres áreas disciplinares mencionadas anteriormente. Algunos consideran que el principal objetivo de una educación STEAM es formar recursos humanos creativos que contribuyan al desarrollo y al progreso científico tecnológico en los sectores de ciencia y tecnología (García Cartagena, Reyes González \& Burgos Oviedo, 2017, Zamorano Escalona, García Cartagena y Reyes González, 2018) y que a su vez, favorecería un educación más inclusiva y la construcción de un conocimiento científico crítico (Coello Pisco, Crespo Vaca, Hidalgo Crespo \& Díaz Jiménez, 2018, Ocaña Rebollo, Romero Albaladejo \& Gil Cuadra, 2017).

Un punto importante a tener en cuenta es que no todo lo que se nombra como STEM hace referencia al enfoque interdisciplinar. En muchos casos, suelen utilizarse las siglas para referirse a un conjunto particular de disciplinas con un enfoque multidisciplinar que suele ir acompañado además, de un recorte en cuanto a la consideración de las disciplinas científicas, limitándolas de manera exclusiva a las naturales y experimentales, y de manera similar, acotando la tecnología al uso de software o a los dispositivos de comunicación (computador, Tablet, móvil) (Rainey, Dancy, Mickelson, Stearns \& Moller, 2018), lo que hoy en día implica un reduccionismo, al menos cuestionable.

Para dar un ejemplo de la relevancia que ha alcanzado en nuestros días el enfoque STEAM en el marco de la interdisciplinariedad, se puede mencionar la carrera de especialista en enseñanza con enfoque STEAM que ofrece la Universidad de Burgos en España, que lo define de la siguiente manera en su folleto promocional: Enfoque educativo interdisciplinar en el que los contenidos de cada disciplina se aprenden de forma integrada, a partir de problemas contextuales planteados de manera significativa para los estudiantes a través de la experimentación científica y la inclusión de programación computacional y robótica educativa.

Las fuentes revisadas en este texto sobre este tema, dan cuenta de la diversidad de propuestas que se engloban en este enfoque. Así por ejemplo, puede haber proyectos STEAM que se implementen como eje vertebrador de una institución, o por el contrario, que se desarrollen en un curso en particular. En cuanto a su duración, existen proyectos anuales hasta proyectos de pocas semanas. Y respecto a las temáticas, pueden ser tan amplias como las civilizaciones mesoamericanas o el cambio climático, o restringirse a una unidad temática particular. De la misma manera, el proyecto puede implementarlo un único docente o un equipo de varios profesores, que a su vez pueden organizarse para trabajar de manera colaborativa, o cada uno ocuparse de una parte en particular sin establecer una interacción explícita con los demás docentes. Dada la naturaleza integrativa de este enfoque, resulta de especial interés para su aplicación principalmente en el nivel primario y en la secundaria básica (aunque no de manera excluyente).

Si se decide implementar este enfoque a modo de innovación en un contexto escolar determinado, es importante tener en cuenta que un cambio en la manera de enseñar condiciona necesariamente, un cambio en la manera de evaluar. Siendo que la principal característica que debe tener una evaluación es la coherencia con la enseñanza realizada, este enfoque no sólo implica tomar decisiones sobre los contenidos a ser incluidos en los exámenes, sino también en cuanto al propio enfoque interdisciplinar y cómo se consideran los diferentes aportes de los actores y disciplinas intervinientes en el desarrollo del proyecto.

El modelo CTS o Ciencia - Tecnología - Sociedad. Este enfoque interdisciplinar para la enseñanza de las cien- 
cias surgió en los Estados Unidos en la década de 1970 (Membiela, 1997) (Figura 1).

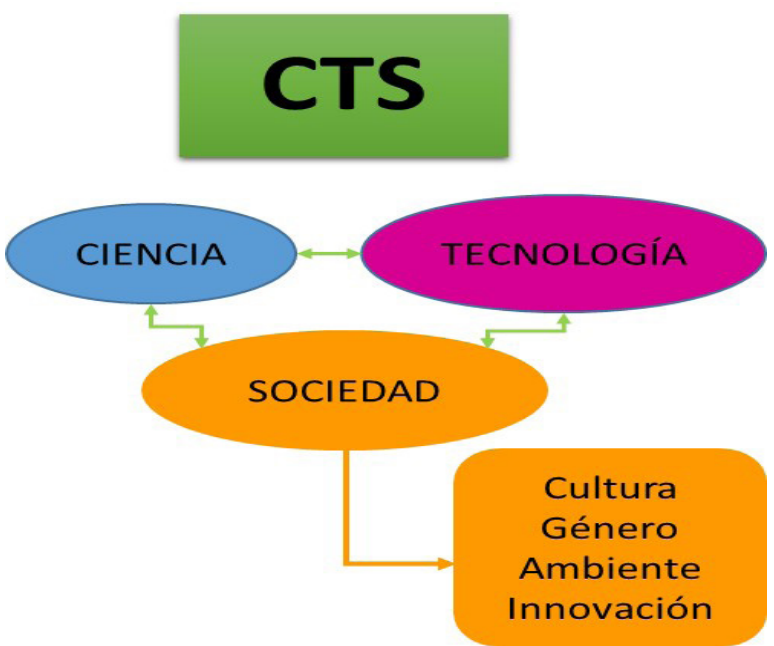

Figura 1. Componentes del enfoque CTS (elaboración propia)

Desde nuestra perspectiva, la $\mathrm{S}$ de sociedad es lo suficientemente amplia para incluir aspectos como la cultura, la problemática de género (Camacho, 2018), nuestras relaciones con el ambiente o el efecto que nos provocan las innovaciones, por lo que nosotros creemos que no es necesario incluir ninguna otra letra. En este sentido, el enfoque CTS es un área de estudios muy amplia que incluye a su vez diversos subconjuntos, muchas veces asociado al concepto de sostenibilidad (o sustentabilidad), que intenta hacer frente a la emergencia planetaria considerando los aportes de la ciencia y la tecnología (Solbes \& Vilches, 1989,2004, Vilches \& Gil Pérez, 2013).

Entre los beneficios asociados a este enfoque se encuentra que "promueve la alfabetización científica y tecnológica de los ciudadanos para que puedan participar en el proceso democrático de toma de decisiones y en la resolución de problemas relacionados con la ciencia y la tecnología." (Membiela, 1997, p. 51).

Por su parte, la Organización de los Estados Iberoamericanos (OEI) (http://www.oei.es/cts.htm) define al movimiento CTS (Membiela \& Padilla, 2005).

como un campo de trabajo bien consolidado institucionalmente en universidades, centros educativos y administraciones públicas de numerosos países industrializados que requiere de un enfoque de índole interdisciplinar, concurriendo en él disciplinas de las ciencias sociales, la tecnología, la sociología del conocimiento científico, la teoría de la educación y la eco- nomía del cambio técnico, en el que se destacan sus objetivos sociales:

- Valorizar en un sentido global la diversidad cultural, social y ambiental, evitando cualquier tipo de discriminación entre las personas.

- Mostrar una imagen académica de ciencia que de cuenta de la naturaleza humana de la actividad científica y por tanto, como un elemento de la cultura de una sociedad,

- Estimular las actitudes y prácticas democráticas para la participación ciudadana en cuestiones vinculadas al desarrollo tecnológico y al medio ambiente.

- Renovar la educación en ciencias naturales y la alfabetización científica de modo de promover la vocación de los jóvenes para su estudio.

En la región iberoamericana, el desarrollo de los estudios CTS es todavía muy reciente (Farré \& Lorenzo, 2019) con escasa proyección social y educativa. En consecuencia, resulta difícil implementar innovaciones en la enseñanza desde este enfoque, ni tampoco recomendable replicar acríticamente las experiencias y modelos de otras latitudes porque su transferencia exige que sean contextualizadas y ajustadas a nuestros escenarios particulares.

\section{La interdisciplinariedad como estrategia para la formación y actualización de profesores}

- El rol de los contenidos en los diseños de enseñanza disciplinares e interdisciplinares. El abordaje de problemáticas reales (en contraposición a los problemas "ideales" propios de la enseñanza de las ciencias) conlleva una gran diversidad de contenidos pertenecientes a diferentes dominios de conocimiento. Podemos intentar describir esta situación apelando a una analogía con la elaboración de cestas de mimbre. Podemos pensar que en los niveles iniciales, a edades tempranas, un abordaje posible sería trabajar de manera interdisciplinaria con la "cesta terminada". A medida que el estudiante vaya avanzando en el sistema educativo, irá "destejiendo la cesta" reconociendo las singularidades de sus diferentes partes, hasta alcanzar el estudio individual de cada una de las "ramitas de mimbre" (especificidad de los contenidos). A los solos fines de ilustrar esta comparación con algún ejemplo, al comienzo se puede comenzar el estudio considerando propiedades extensivas (tamaño, masa, forma) para finalizar con el análisis de la 
flexibilidad de la ramita de mimbre, o su composición química.

- Tal vez sería recomendable, pensar en una diagramación general a modo de "pulsos" donde se plantee periódicamente y de manera complementaria, un ida y vuelta desde lo general a lo particular, de lo concreto a lo abstracto y así con cada uno de los criterios que pueden plantearse en la organización curricular. En consecuencia, el trabajo de selección y secuenciación de los contenidos (Lorenzo, 2018) para este tipo de propuesta adquiere una particular relevancia, porque exige la toma de decisiones consensuada por parte del profesorado $y$ de las autoridades educativas.

La vinculación de las prácticas docentes con la investigación en didáctica de las ciencias. Una alternativa potente para la capacitación de los profesores es involucrarlos con los procesos y productos de la investigación (Nussbaum, 2017). La investigación en didáctica de las ciencias ha generado muchos conocimientos a lo largo de los últimos cuarenta años. Sin embargo, su impacto sobre las transformaciones de las prácticas de enseñanza suele ser bajo y por eso se reclama un mayor grado de transferencia a la investigación científica para lo cual se generan proyectos para el desarrollo de soluciones a problemas específicos. En este sentido, es importante que los profesores de aula, se acerquen y se apropien de los productos de la ciencia, es decir, de los resultados de las investigaciones, sobre todo de aquellas que han sido desarrolladas en nuestra región. Esta sería una primera manera de contactarse con la investigación, actuando como consumidores críticos.

Otra forma de participación posible, es involucrarse en el desarrollo de una investigación, actuando así como productores de conocimiento. De ninguna manera, esta segunda opción debería ser considerada como mandatoria para la totalidad del profesorado. Muy por el contrario, la incorporación de los profesores al mundo de la investigación debe partir de una disposición personal y voluntaria, que le permita analizar opciones acerca de posibles líneas y grupos de investigación con quienes relacionarse. Esto ofrece la oportunidad de desarrollar investigaciones basadas en diseño que en la mayoría de los casos recurren a un enfoque interdisciplinar para la solución del problema en cuestión (de Benito Crosetti, \& Salinas Ibáñez, 2016, Godino et al. 2013, Molina et al., 2011, Silva-Weiss, Pérez-Lorca \& Quiroz, 2019, Valverde-Berrocoso, 2016).

Tanto si se trata de una nueva propuesta para la enseñanza como de un proyecto de innovación, implica acercarse al aula de ciencias con una mirada interdisciplinar que contemple la epistemología, la historia, la sociología y la filosofía de las ciencias, para construir una idea de naturaleza de la ciencia como un conjunto de contenidos metacientíficos con valor para la educación científica (Adúriz Bravo, 2011, Lederman, Antink \& Bartos, 2012).

\section{Un ejemplo de práctica para un enfoque innovador de enseñanza}

Tomando el enfoque STEM, se presenta la actividad denominada "Preparación de sales perfumadas" (Lorenzo \& Cortez Iribarren, 2018). Esta se basa en el modelo de actividades experimentales simples -AES- (Reverdito \& Lorenzo, 2007), es decir una actividad que puede realizarse en cualquier escenario educativo, con materiales seguros y de bajo costo como se muestra en la Figura 2.

Resumidamente, la experiencia consiste en preparar una mezcla en base a productos fácilmente asequibles, como el colorante vegetal, sal de cocina y alguna esencia natural. El resultado, una sal colorida que puede perfumar un ambiente o ser utilizada para el baño.

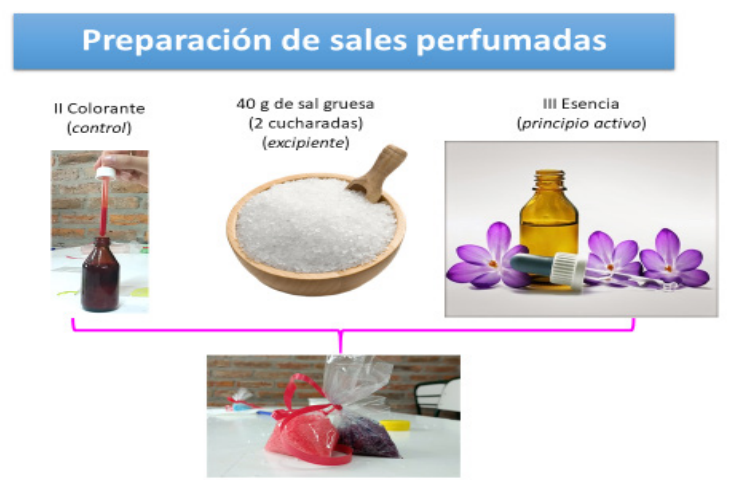

Figura 2. Ingredientes para la preparación de sales perfumadas (tomado de Lorenzo \& Cortez Iribarren, 2018).

Un análisis de la actividad, permite detectar el uso del colorante como un elemento que garantiza el correcto proceso de mezclado, asegurando la distribución de la esencia en todo el producto. Fácilmente pueden reconocerse muchos contenidos propios del campo de las ciencias naturales, tales como: propiedades de los materiales, sistemas materiales, homogéneos y heterogéneos, mezclas y sustancias, solubilidad, por mencionar algunos.

Las esencias pueden adquirirse en cualquier tienda de repostería, o también pueden obtenerse a partir de 
una extracción, planteando una experiencia de complejidad media en un laboratorio de química, como se muestra en la Figura 3, en el marco de un curso para profesores que ofrecimos hace algunos años.

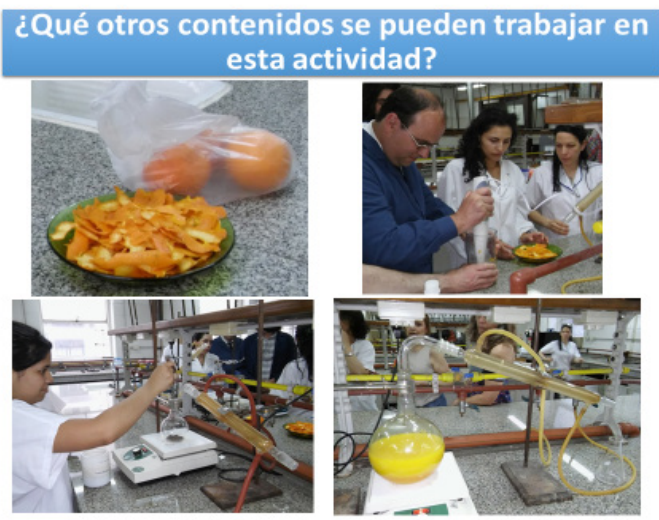

Figura 3. Extracción de esencia de naranja (fotografías tomadas en el laboratorio de Química Orgánica, FFYBUBA).

Otros tópicos que pueden abordarse a partir de esta experiencia, están vinculados a temas de alimentos, nutrición y salud. Por ejemplo, el uso de sal como conservante de alimentos desde la antigüedad, la participación del catión sodio en distintos pasos del metabolismo humano y sus efectos en la presión cardíaca. También, pueden trabajarse con un enfoque sociocultural, reconociendo el uso de las sales como mordientes, o el uso de colorantes en las tinturas de telas o en la composición de pinturas. Y podrían pensarse muchos más...

\section{Reflexiones finales}

La interdisciplinariedad es un enfoque que provee un espacio adecuado para la construcción de conocimiento, tanto en el ámbito escolar como en la investigación científica.

En cuanto a la investigación científica, el trabajo interdisciplinar está teniendo un desarrollo cada vez más importante al abordar diferentes objetos de investigación de manera holística y multidimensional. Este debe garantizar la comunicación de sus resultados tanto en los ámbitos académicos como escolares y sociales ya que contribuyen a la reflexión sobre las prácticas educativas.
No hemos considerado en este texto, el rol de los contextos no formales (Guisasola \& Morentin, 2007), ni la importancia de los lenguajes en la enseñanza de las ciencias (Lemke, 2002, Quílez, 2016). Tampoco hemos considerado las potencialidades de propuestas interdisciplinares con áreas como la geografía o la economía. No obstante, hemos intentando mostrar a traves de un ejemplo concreto para el área de química, las diversas alternativasde abordajes posibles, cada una de las cuales puede desarrollarse con diferentes niveles de profundidad de acuerdo con el nivel educativo en el que se apliquen.

Los enfoques interdisciplinares, como CTS y STEAM, brindan la oportunidad de aproximarse a contenidos del campo de las ciencias naturales de manera significativa y mejorar las competencias científicas, al considerar la participación ciudadana en la resolución de problemáticas sociocientíficas (Pro-Bueno \& Ezquerra, 2005, Ruiz, Solbes \& Furió, 2013, Solbes \& Torres, 2014).

Los intersticios y las posibles imbricaciones entre diferentes dominios de conocimiento son prácticamente inacabables, por eso pueden surgir nuevas realaciones entre áreas disciplinares que hasta ahora se han mantenido alejadas. Cabe resaltar por lo tanto, la gran importancia del trabajo del profesor en el diseño y planificación de la propuesta de actividades para sus estudiantes, el cual nunca podrá ser delegado en nada ni nadie.

Para finalizar, la interdisciplinariedad nos plantea nuevos desafíos para superar las tensiones entre los distintos dominios de conocimiento con el fin de establecer los consensos necesarios para acordar un lenguaje común que favorezca el diálogo y la comunicación entre las diferentes áreas disciplinares. Consecuentemente, se hace necesario generar los espacios personales e institucionales propicios para el desarrollo de proyectos de investigación educativa desde una perspectiva interdisciplinar, promoviendo la formación de los profesores en servicio para el desarrollo de prácticas de enseñanza desde el enfoque interdisciplinar y también, conformar equipos de trabajo coherentes con este enfoque.

\section{Agradecimientos}

Proyectos de Investigación: UBACYT-201820020170100448BA, ANPCYT-FONCYT PICT-20150044, CONICET PIP11220130100609CO. 


\section{Referencias bibliográficas}

Adúriz Bravo, A. (2011). Desde la enseñanza de los "productos de la ciencia" hacia la enseñanza de los "procesos de la ciencia" en la Universidad. Cuadernillos de actualización para pensar la Enseñanza Universitaria,6 (3). Universidad Nacional de Río Cuarto.

Bolarín Martínez, M. J., Moreno Yus, A. \& Porto Currás, M. (2013). Coordinación docente e interdisciplinariedad: análisis de su contribución a la adquisición de competencias docentes y discentes, Revista de Docencia Universitaria, 11(2), 443-462.

Camacho, J. (2018). Identificación y caracterización de las creencias de docentes hombres y mujeres acerca de la relación ciencia - género en la educación científica. Estudios Pedagógicos, [S.I.], 43(3), 63-81. Recuperado de http://revistas.uach.cl/index.php/ estped/article/view/1234

Carvajal Escobar, Y. (2010). Interdisciplinariedad: desafío para la educación superior y la investigación, Revista Luna Azul, 31, 156-169.

Castañer Ballcells, M. \& Trigo Aza, E. (1995). La interdisciplinariedad en la Educación secundaria obligatoria: propuestas teórico-prácticas. Zaragoza: Inde Publicaciones.

Coello Pisco, S., Crespo Vaca, T., Hidalgo Crespo, J. \& Díaz Jiménez, D. (2018). El modelo STEM como recurso metodológico didáctico para construir el conocimiento científico critico de estudiantes de Física, Latin-American Journal of Physics Education, 12 (2), 2306. Recuperado de http://www.lajpe.org/

de Benito Crosetti, \& Salinas Ibáñez, 2016, Godino et al. 2013, Molina et al., 2011, Silva-Weiss, Pérez-Lorca \& Quiroz, 2019, Valverde-Berrocoso, 2016).

de Benito Crosetti, B., \& Salinas Ibáñez, J. M. (2016). La Investigación Basada en Diseño en Tecnología Educativa. Revista Interuniversitaria De Investigación En Tecnología Educativa. https://doi.org/10.6018/ riite2016/260631 (https://revistas.um.es/riite/article/ view/260631)

Farré, A. \& Lorenzo, M. G. (2019). El enfoque CTS en la formación de profesores de ciencia. Estrategias de enseñanza de la naturaleza de la ciencia y la tecnología, Boletín de la AIA-CTS, 09 (1), 31-36.Recuperado de http://aia-cts.web.ua.pt/wp-content/uploads/2019/04/ AIA-CTS Boletim09.pdf

García Cartagena, Y., Reyes González, D. \& Burgos Oviedo, F. (2017). Actividades STEM en la formación inicial de profesores: nuevos enfoques didácticos para los desafíos del siglo XXI. Revista Electrónica Diálogos Educativos, 17 (33), 35-46. Recuperado de http://revistas.umce.cl/ index.php/dialogoseducativos/article/view/1168/1177

Godino, J., Batanero, C., Contreras, A., Estepa, A., Lacasta, E. y Wilhelmi, M. (2013). La ingeniería didáctica como investigación basada en el diseño. Recuperado de http://enfoqueontosemiotico.ugr.es/documentos/ JDGodino 2013 Ingenieria didactica.pdf

Guisasola, J. \& Morentin, M. (2007). ¿Qué papel tienen las visitas escolares a los museos de ciencias en el aprendizaje de las ciencias? Una revisión de las investigaciones. Enseñanza de las Ciencias, 25(3), 401414.

Jones, M. G., Gardner, G. E., Robertson, L. \& Robert, S. (2013). Science Professional Learning Communities: Beyond a singular view of teacher professional development. International journal of science education, 35(10), 1756-1774. https://doi.org/10.1080 09500693.2013 .791957

Lantz-Andersson, A., Lundin, M. \& Selwyn, N. (2018). Twenty years of online teacher communities: A systematic review of formally-organized and informally-developed professional learning groups. Teaching and Teacher Education, 75, 302-315. https://doi.org//10.1016/j. tate.2018.07.008

Lederman, N. G., Antink, A., \& Bartos, S. (2012). Nature of Science, Scientific Inquiry, and Socio-Scientific Issues Arising from Genetics: A Pathway to Developing a Scientifically Literate Citizenry, Science y Education, 23 (2), 285-302.

Lemke, J. (2002) Enseñar todos los lenguajes de la ciencia: palabras, símbolos, imágenes y acciones. En: Benlloch, M. (comp.) La educación en ciencias: ideas para mejorar su práctica. Barcelona: Paidós.

Lenoir,Y.(2013)Interdisciplinariedadeneducación:unasíntesis de sus especificidades y actualización. INTERdisciplina, [S.I.], 1(1), 51-86 Recuperado de http://revistas.unam. $\underline{\mathrm{mx} / \text { index.php/inter/article/view/46514/41769 }}$

Llano Arana, L., Gutiérrez Escobar, M., Stable Rodríguez, A., Núñez Martínez, M., Masó Rivero, R. \& Rojas Rivero, B. (2016). La interdisciplinariedad: una necesidad contemporánea para favorecer el proceso de enseñanza aprendizaje. MediSur, 14(3), 320-327. Recuperado de http://scielo.sld.cu/scielo.php?script=sci arttext\&pid=S1727-897X2016000300015\&Ing=es\&tlng= es.

Lorenzo, M. G. \& Cortez Iribarren, C. (2018). Preparación de sales perfumadas como estrategia didáctica desde una perspectiva química, Revista Educación en la Química, 24 (1), 37-47.Recuperado de http://www.adeqra.com.ar

Lorenzo, M. G. (2018). Los contenidos de ciencias naturales en la enseñanza universitaria: especificidad, abstracción y orientación profesional, Aula Universitaria, 19, Recuperado de https://doi.org/10.14409/au.v0i19

Membiela, P. (1997). Una revisión del movimiento educativo ciencia-tecnología-sociedad. Enseñanza de las Ciencias, 15(1), 51- 57. 
Molina, M., Castro, E., Molina, J. L. y Castro, E. (2011). Un acercamiento a la investigación de diseño a través de los experimentos de enseñanza, Enseñanza de las ciencias: revista de investigación y experiencias didácticas, [en línea], 29(1),75-88, Recuperado de https://www.raco. cat/index.php/Ensenanza/article/view/243824

Membiela, P. y Padilla, Y. (Eds.) (2005). Retos y perspectivas de la enseñanza de las ciencias y el enfoque CienciaTecnología-Sociedad en los inicios del siglo XXI. España: Educación Editora. Recuperado de: http:// aia-cts.web.ua.pt/wp-content/uploads/2013/07/ RetosyperspectivasCTS.pdf

Nussbaum, L. (2017). Investigar con docentes. En Moore, E. y Dooly, M. (Eds). Enfoques cualitativos para la investigación en educación plurilingüe (pp. 23-45). Research-publishing.net.https://doi.org/10.14705/ rpnet.2017.emmd2016.620

Ocaña Rebollo, G., Romero Albaladejo, I. \& Gil Cuadra F. (2017). Educación STEM para integrar conocimientos científicos en la asignatura "tecnología industrial" de bachillerato, Enseñanza de las Ciencias, № extraordinario, 5327-5333.

Olivé Morett, L., Argueta Villamar, A. \& Puchet Anyul, M. (2018). Interdisciplina y transdisciplina frente a los conocimientos tradicionales, Revista CTS, 38 (13), 135153.

Palma de Arraga, L. (1998). Fortalecimiento de la capacidad interdisciplinaria en educación ambiental. Revista Iberoamericana De Educación, 16, 65-99. https://doi. org/10.35362/rie1601112

Peñuela Velásquez, A. (2005). La transdisciplinariedad: Más allá de los conceptos, la dialéctica. Andamios, 1(2), 43-77. Recuperado de http://www.scielo.org. $\mathrm{mx} /$ scielo.php?script $=\mathrm{sci}$ arttext \&pid=S1870$00632005000300003 \& \operatorname{lng}=$ es\&tlng=es.

Posada Álvarez, R. (2004). Formación superior basada en competencias, interdisciplinariedad y trabajo autónomo del estudiante. Revista Iberoamericana De Educación, 35(1), 1-33. https://doi.org/10.35362/ rie3512870

Pro-Bueno, A. D. \& Ezquerra, A. (2005). ¿Qué ciencia ve nuestra sociedad? Alambique: Didáctica de las Ciencias Experimentales, 12(43), 37-48.

Quílez-Pardo, J. (2016) ¿Es el profesor de química también profesor de Lengua? Educación química, 27, 105-114.

Rainey, K., Dancy, M., Mickelson, R., Stearns, E. \& Moller, $S$. (2018). Race and gender differences in how sense of belonging influences decisions to major in STEM, International Journal of STEM Education, 85 (10), https://doi.org/10.1186/s40594-018-0115-6
Reverdito, A. \& Lorenzo, M. G. (2007). Actividades experimentales simples. Un punto de partida posible para la enseñanza de la química, Educación en la Química, 13(2), 108-121.Recuperado de http://www. adeqra.com.ar

Ruiz, J., Solbes, J. \& Furió, C. (2013). Debates sobre cuestiones sociocientíficas. Una herramienta para aprender física y química. Textos de Didáctica de la Lengua y de la Literatura, 64, 32-39.

Santillán Aguirre, J. P., Cadena Vaca, V. del C., \& Cadena Vaca, M. (2019). Educación Steam: entrada a la sociedad del conocimiento. Ciencia Digital, 3(3.4.), 212-227. https:// doi.org/10.33262/cienciadigital.v3i3.4.847

Silva-Weiss, A., Pérez-Lorca, A., y Quiroz, M. (2019). Investigación basada en diseño para la mejora sostenida del aprendizaje auténtico. Revista de Gestión de la Innovación en Educación Superior REGIES 4, pp. 07-33.

Solbes, J. \& Torres, N. (2014). Aspectos convergentes del pensamiento crítico y las cuestiones sociocientíficas. Góndola, Enseñanza y Aprendizaje de las Ciencias, 9(1), 1-13.

Solbes, J. \& Vilches, A. (1989). Interacciones ciencia/técnica/ sociedad: un instrumento de cambio actitudinal. Enseñanza de las Ciencias, 7(I), 14-20.

Solbes, J. \& Vilches, A. (2004). Papel de las relaciones entre ciencia, tecnología, sociedad y ambiente en la formación ciudadana. Enseñanza de las Ciencias, 22(3), 337 -348.

Valverde-Berrocoso, J. (2016). La investigación en Tecnología Educativa y las nuevas ecologías del aprendizaje: DesignBased Research (DBR) como enfoque metodológico. RIITE. Revista Interuniversitaria de Investigación en Tecnología Educativa, 0, 60-73. http://doi.org/10.6018/ $\underline{\text { riite/2016/257931 }}$

Vilches, A. \& Gil Pérez, D. (2013). Ciencia de la sostenibilidad: Un nuevo campo de conocimientos al que la química y la educación química están contribuyendo, Educación Química, 24(2), 199-206. Recuperado de http:// aia-cts.web.ua.pt/wp-content/uploads/2014/02/ VilchesGil2013 CS EQ.pdf

Zamorano Escalona, T., García Cartagena, \&. y Reyes González, D. (2018). Educación para el sujeto del siglo XXI: principales características del enfoque STEAM desde la mirada educacional, Contextos, 41.Recuperado de http://revistas.umce.cl/index.php/contextos/article/ view/1395 